\title{
Urachal cyst
}

INSERM

\section{Source}

INSERM. (1999). Orphanet: an online rare disease and orphan drug data base. Urachal cyst. ORPHA:488

Urachal cyst is a congenital urachal anomaly (see this term) characterized by a failure of complete closure of the urachus, in which both ends are closed but the central lumen remains patent. It is typically asymptomatic but may become clinically sig nificant when infected, presenting as a mass in the umbilical region accompanied by abdominal pain and fever. 